Виконано аналіз принципів SMM (Social Media Marketing) на придатність для просування наукового контенту. Побудована модель життевого ииклу публікацій на основі ланияога Маркова. Досліджено особливості застосування методології SMM за допомогою розробленої марківської моделі. Показано, що супровід авторами публікаиій в соціальних мережах створюе умови для успішного цитування

Ключові слова: SMM, наукометрія, соціальні мережі, діджиталізація, цифровий контент, ResearchGate, DOI

$$
\text { 口- }
$$

Выполнен анализ принципов SMM (Social Media Marketing) на применимость для продвижения научного контента. Построена модель жизненного цикла публикаций на основе цепи Маркова. Исследованы особенности применения методологии SMM с помощью, разработанной марковской модели. Показано, ито сопровождение авторами публикаций в социальных сетях создает условия для успешного цичтирования

Ключевые слова: SMM, наукометрия, сочиальные сети, диджитализация, цифровой контент, ResearchGate, DOI

\section{COMMUNICATION MANAGEMENT IN SOCIAL NETWORKS FOR THE ACTUALIZATION OF PUBLICATIONS IN THE WORLD SCIENTIFIC COMMUNITY ON THE EXAMPLE OF THE NETWORK RESEARCHGATE}

K. Kolesnikova Doctor of Technical Sciences, Assistant Professor Department of Information technology in mechanical engineering*

D. L u kia nov $\mathrm{PhD}$, Assistant Professor**

V. Goguns k i i

Doctor of Technical Sciences, Professor Department of Systems Management Life Safety*

E-mail:vgog@i.ua

V. I a kovenko

Postgraduate student

Company "Tobii Technology" AB

Karlsrovägen, 2D, Danderyd, Stockholm, Sweden, 18253

G. O borska

$\mathrm{PhD}$

Advertising agency "Formula uspecha" Velyka Arnautska str., 76-a, Odessa, Ukraine, 65000

A. Negri

Postgraduate student «INTERNETSTORES» GmbH

Fritz-Müller-Straße, 106-108, Esslingen am Neckar, Germany, 73730

A. Koly ad a

$\mathrm{PhD}$

GmBH Luxoft UA

Kartamyshevskaya str., 9V, Odessa, Ukraine, 65091

K. D m i t re n ko

Engineer

Department of Systems Management Life Safety*

T. O l e k h

$\mathrm{PhD}$, Assistant Professor Department of higher mathematics and simulation systems*

K. B e s pa n sk a y - P a u le n k a

$\mathrm{PhD}$, Assistant Professor**

*Odessa National Polytechnic University

Shevchenko ave., 1, Odessa, Ukraine, 65044

** Department of General and Clinical Psychology

Belarusian State University

Nezavisimosti ave., 4, Minsk, Belarus, 220030

\section{Introduction}

Dissemination of the outcomes of research and scientific activity has been recently undergoing major changes in con- nection with the Internet development. Social networks are emerging and developing. Specialization of social networks is formed - from the placement of graphical content, such as in Instagram [1], to the placement of scientific content, such 
as in ResearchGate [2]. Moreover, in each of these segments, there is an increase in competition, as well as "mergers and acquisitions". An example of this is the acquisition of Mendeley system [3] by the company Elsevier [4], data integration between SCOPUS and ORCID [5], etc. The world is rapidly becoming "digitized".

The relevance of research is driven by the need to promote scientific ideas in the environment of the world scientific community [6]. In the digital era, methods and tools for the promotion of digital content proved its effectiveness in a world of fierce competition. That is why understanding and ability to use these methods will make it possible to help overcome the isolation of individual researchers and research teams through scientific communication, adopted worldwide [6]. One of such tools is the methodology of SMM (Social Media Marketing) - marketing with the use of social networks.

\section{Literature review and problem statement}

The world scientific community recognized the concept on that one of the main results of scientific activity is the scientific articles, reflecting outcomes of theoretical and experimental research [6]. In this case, it is accepted to estimate the level of significance of scientific contribution to development of theory and practice by using the articles' citation indices [7]. Indeed, citation of certain articles by colleagues in their publications is, as a rule, a positive assessment of the cited articles [8]. Existence of social networks of scientists on the World Web creates conditions for wider awareness of the global community about results of scientific research [9]. World practice of communications of scientists' community through scientific publications on the World Web testifies to the expediency of such systems and information technologies [10]. After all, it is difficult to deny the obvious fact that those publications are cited, which are accessible to a wide circle of readers. That is, for a certain article to be cited, it is necessary that the article should be read by as large number of specialists and scientists as possible [6].

The industry of scientific journals publishing in the form of paper copies currently focuses on the duplication and presentation of printed publications in the form of digitized copies. The process of "digitizing" the scientific content, with the help of social scientific networks, makes it possible to distribute worldwide many clones of the primary message. "Publish or Perish" is the name the authors of [10] gave to their search engine [10]. In translation, it sounds like a warning. In other words, authors of publications should promote their scientific content, or else they would lose the competition of scientific ideas.

Promotion of publications to readers in the Internet space is connected with active participation of authors when submitting publications to such information systems as ResearchGate [2], Mendeley [3], ORCID [5], Google Scholar [8], and Academia [9]. Naturally, maintaining libraries of publications in these systems is rather time-consuming. However, such an approach is justified - no one but the author can objectively present scientific findings.

The relevance of the issue of information communications involves promotion of scientific ideas and timely exchange of research results [4]. At the same time, journal publications on the pages of even highly reputable journals are printed with limited circulation [6]. Therefore, a com- bination of approaches of the SMM methodology (Social Media Marketing) and the tasks of promotion of scientific content on the Internet determines scientific novelty of the present research. In such context, this problem was not addressed in scientific publications by other scientists. As a rule, they consider either scientometric indicators [10] or the problems in content identification to detect texts match [11]. The use of modern Internet technologies, presented by software products for the social networks ResearchGate [2], Mendeley [3], Google Scholar [8], Academia [9], and Publish or Perish [10], creates conditions for a significant expansion of the audience of readers of scientific articles. Democratic social network Internet resources allow overcoming distances, bring together researchers working in close scientific directions, remove barriers between a student and an academician - they are the future for development of scientific relations in a world scientific community [6].

\section{The aim and objectives of the study}

The goal of present research is to generalize and develop applied aspects in using the existing social scientific networks on the example of the network ResearchGate [2] for the promotion of scientific publications in scientific environment. This will expand the audience of readers of scientific articles and ultimately lead to an increase in the citation indices of papers.

To accomplish the set goal, the following tasks had to be solved:

- to design a life-cycle model for scientific publications based on the Markov chain;

- to reproduce and implement in the domain of social scientific communications the experience of best practices of SMM (Social Media Marketing) [12];

- to explore practical aspects in the application of a SMM methodology employing the designed Markov model.

\section{Designing a life-cycle model of a publication}

As it is known, the life cycle of publications consists of the following stages: carrying out research - preparation of a manuscript - editorial preparation of an article - production of a printed (electronic) journal - submitting a journal to scientometric databases - waiting for a reader [13]. At the last stage, "an independent life" of a publication begins. The scientific community gets a chance to get acquainted with the content of the article.

After an article is published, the authors of a publication are no longer involved in the process of promotion of an article and cannot actively influence the process of their work being cited by other authors. That is why we can offer the main recommendation: articles should be published in professional journals where colleagues can get acquainted with them and appreciate them by citing [13].

To model the phase of an "independent life" of articles, we will develop a Markov chain, which will make it possible to represent a random process of citing articles. Let us designate as $S_{i}\{i=1,2, \ldots 5\}$ the possible states (relationships) of readers' community to scientific publications at this stage: $S_{1}$ - Awareless (unawareness); $S_{2}-$ Awareness (awareness); $S_{3}$ - Attitude (positive attitude); $S_{4}-$ Action (citing); $S_{5}-$ Abort (negative attitude). 
The specified conditions characterize cohort distribution of potential readers of an article by the level of assessment of the article [13]. That is why the given states of the proposed model 5A's constitute a complete group of events, in which one state will definitely be implemented.

Transitions between the states are shown in the marked graph (Fig. 1). State $S_{5}-$ Abort (negative attitude) is particularly positioned in the Markov chain. The system passes into this state after state $S_{2}$, to which it is possible to return after a more detailed study of the publications. At the same time, negative attitude towards an article does not rule out the probability of its citation, as shown on the graph by an arrow of transition from $S_{5}$ to $S_{4}$. In addition, transition from state $S_{5}$ to $S_{1}$ is possible, due to the "forgetting" processes by Ebbinghaus [14].

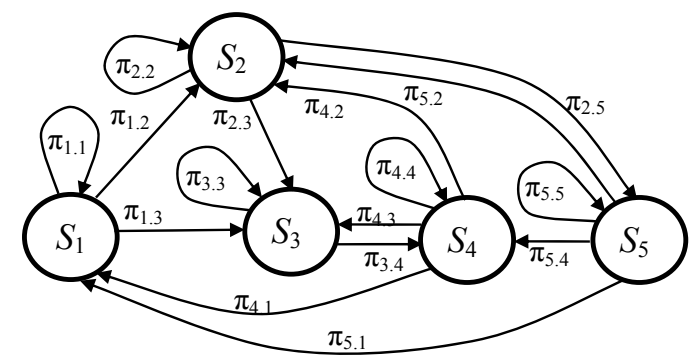

Fig. 1. Graph of states of the model 5A's: $S_{1}-$ unawareness; $S_{2}-$ awareness; $S_{3}-$ positive attitude; $S_{4}-$ citing; $S_{5}$ - negative attitude

In model 5A's, a random process of changing $S_{i}\{i=$ $=1,2, \ldots 5\}$ over time is implemented. Time $t[0, \mathrm{~T}]$ runs discrete series of values of $t_{0}, t_{1}, t_{2}, \ldots, t_{N}:\left\{t_{n}, n=\overline{0, N}\right\}$, and random magnitude $S_{i}\left(t_{n}\right)=\left.S_{i}\right|_{n}$ can take a discrete set of values. A "markovian essence" of scientific communications is proved by the fact that both in communications and in the Markov chains, there may be changes in the probabilities of states of the system by steps $k=n$. There exist the probabilities of transitions to other states [15]. The sum of transition probabilities from a certain state is equal to unity [16]. The sum of probabilities of all states at every step is also equal to unity [17]. There is a similarity of a topological structure of transitions [18].

Probabilities of states of the system $\left\{p_{1}(k), p_{2}(k), \ldots, p_{5}(k)\right\}$ change by steps $k$. In this notation of a uniformed model, transition probabilities $\pi_{i j}$ do not depend on a step number. For any step $k$, there are also probabilities of a system delay in the given state. On the graph, the arrows are marked only for the transitions whose transition probabilities are different from zero. "Delay probabilities" $\pi_{i i}$ complement to unity the sum of transitional probabilities by all transitions from the given state [19]. Matrix of transition probabilities of the system takes the form:

$$
\left\|\pi_{i . j}\right\|=\left\|\begin{array}{lllll}
\pi_{1.1} & \pi_{1.2} & \pi_{1.3} & 0 & 0 \\
0 & \pi_{2.2} & \pi_{2.3} & 0 & \pi_{2.5} \\
0 & 0 & \pi_{3.3} & \pi_{3.4} & 0 \\
\pi_{4.1} & \pi_{4.2} & \pi_{4.3} & \pi_{4.4} & 0 \\
\pi_{5.1} & \pi_{5.2} & 0 & \pi_{5.4} & \pi_{5.5}
\end{array}\right\| .
$$

The known general iterative solution to the system of equations of the Markov chain allows us to find probabilities of states $\left\{p_{1}(k), p_{2}(k), \ldots, p_{5}(k)\right\}$ after any $k$-th step [15]:

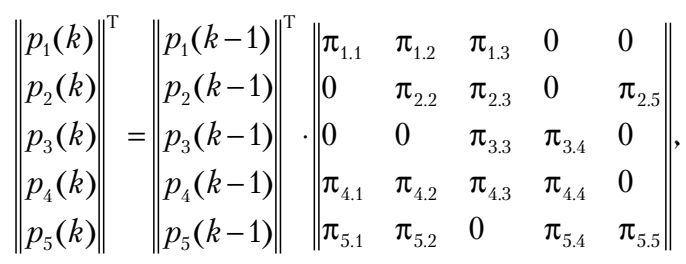

where $\mathrm{T}$ is the transposition mark.

Probabilistic essence of communication processes is represented in the coordinates of totality of probabilities of states $\left\{p_{1}(k), p_{2}(k), \ldots, p_{5}(k)\right\}$ by steps $k$ - contacts that constitute the communication base with changing reader's attitudes to publications. Model 5A's describes such probabilities of states of a complete group of events: $p_{1}(k)$ - unawareness; $p_{2}(k)$ - awareness; $p_{3}(k)$ - positive attitude; $p_{4}(k)$ - citing; $p_{5}(k)$ - negative attitude.

To determine the elements of matrix (1), we use a method of expert evaluation of time cost required for performing certain communications [15]. Transition probabilities $\pi_{s j}\{s \in 1, \ldots, 5 ; j \in 1, \ldots, 5\}$ "set" the Markov chain to representation of properties of a particular object. In this case, to determine transition probabilities $\pi_{s}$, the experts are engaged, who, based on their experience, perform assessment of the values of transition probabilities [19]. It is also possible to use the survey method in order to calculate the values of transition probabilities. Paper [15] proposes a method when values $\pi_{s j}$ are determined based on the characteristics of communications, taking into account time cost, required to perform operations.

Block diagram of communications in the scientific community is represented on the directed graph of the Markov chain (Fig. 1). For any discrete state $s\{s \in 1, \ldots, 5\}$, it is possible to represent the total time $T_{s}$ of communications with other states as the sum of duration of communications with given states $t_{s j}\{s \in 1, \ldots, 5 ; j \in 1, \ldots, 5[15]$ :

$$
T_{s}=\sum_{j=1}^{n=5} t_{s j}
$$

where $t_{s j}$ is the time in communication $s \rightarrow j$ from state $s$.

In each communication $s \rightarrow j$, system can stay for some time $t_{s j}$. Values $\pi_{s j}=t_{s j} / T_{\mathrm{s}}$ make sense of conditional probability of transition to communications $s \rightarrow j$. That is why the sum of all transition probabilities considering (3) for any state $s$ is equal to unity:

$$
\sum_{j=1}^{n=5} \pi_{s j}=\sum_{j=1}^{n=5} \frac{t_{s j}}{T_{s}}=\frac{1}{T_{s}} \sum_{j=1}^{n=5} t_{s j}=1 .
$$

Varying of values of $\pi_{s j}$ enables changing characteristics of the system. The distinctive feature of this approach is that the chain, proposed by Markov, adjusts itself to different possible states of the readers' community by a publication assessment by setting values of $\pi_{s j}$. Transition probabilities for particular states are selected based on expert evaluation from Table 1.

Table 1

Determining the values of transition probabilities $\pi_{s j}$

\begin{tabular}{|c|c|}
\hline $\begin{array}{c}\text { Character of communication } s \rightarrow j \text { by } \\
\text { time cost }\end{array}$ & Transition probabilities $\pi_{s j}$ \\
\hline Maximum time cost & $0.62-1.0$ \\
\hline Medium time cost & $0.38-0.62$ \\
\hline Insignificant time cost & $0.05-0.38$ \\
\hline Minimum time cost & $0-0.05$ \\
\hline
\end{tabular}


As an example, we will consider substantiation of values $\pi_{s}$ for state $S_{3}$ "positive attitude". Conditional transition probability $\pi_{3.4}=0,3$ was accepted in the interval of insignificant time cost. At the same time, the sum of transition probabilities (in a line) for a certain state equals to unity. That is why $\pi_{3.3}=0.7$ - this corresponds to the interval of maximum time cost. Similar assessments were performed for all other states. The following matrix of numerical values for (2) was retrieved:

$$
\left\|\pi_{i j}\right\|=\left\|\begin{array}{lllll}
0,97 & 0,02 & 0,01 & 0 & 0 \\
0 & 0,60 & 0,10 & 0 & 0,30 \\
0 & 0 & 0,70 & 0,30 & 0 \\
0,05 & 0,20 & 0,30 & 0,45 & 0 \\
0,10 & 0,10 & 0 & 0,05 & 0,75
\end{array}\right\| .
$$

Modelling with the use of the designed Markov model for the basic variant of system (3), that is, for the existing situation, showed results that are presented in Fig. 2.

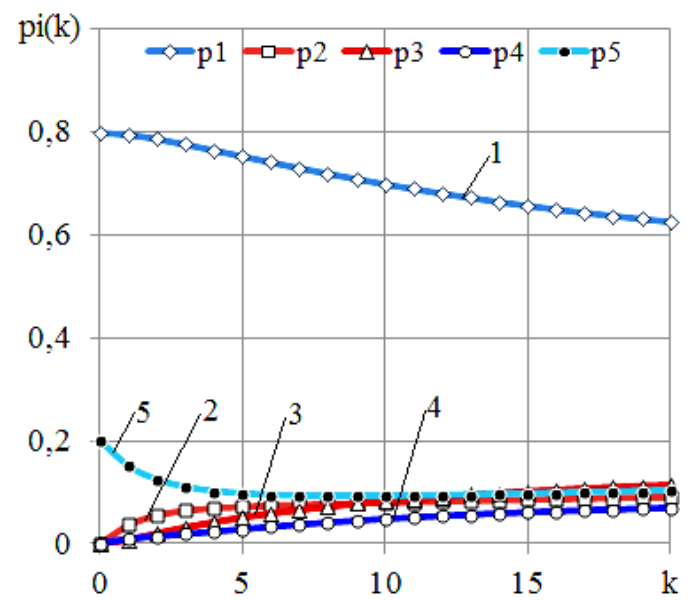

Fig. 2. A change in the probabilities of states of scientists' distribution by the level of attitude to a publication:

1 - unawareness; 2 - awareness; 3 - positive attitude; 4 -citing; 5 - negative attitude

Existing situation in the field of scientific communications for the accepted conditions for participation of the authors of present research in the social network ResearchGate is not effective (Fig. 2). Probability of publications citing is $\mathrm{p}_{4}(20) \approx 0.1$. And the vast majority of articles are in the mode "unawareness", as $p_{1}(20)>0.6$. Therefore, it is necessary to stop waiting passively until results improve and start managing scientific communications.

\section{Communication management for the actualization of publications in the world scientific community}

Based on data [12], postulating by the practical outcomes five key principles for successful promotion of information resources, we will use a technique of analogy and apply these principles in relation to the promotion of outcomes of scientific research.

Principle 1. "One in the field is not a warrior".

The authors of [12] note that it is a team that usually works on a project (Fig. 3). Nobody will see good content without promotion.

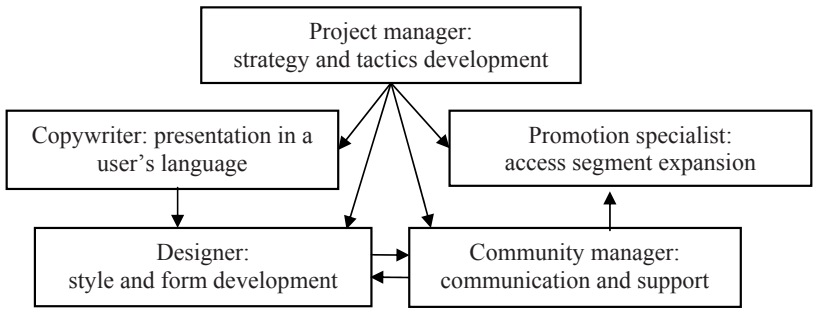

Fig. 3. Structure of SMM direction in the LeadMachine [12]

Of course, virtually every prestigious peer-reviewed journal performs almost all the above functions. On the other hand, it is necessary to understand that "One in the field is not a warrior" already fully applies to individual scientists. In future, advantage will belong to scientific schools, able to be united by the principle of project teams in the process of working on scientific projects, $\mathrm{PhD}$ research, etc. [16]. In the commercial world of information content on the Internet, they say: "Nobody will see good content without promotion" [12]. This statement is also true and for promotion of scientific content. Where and how to promote information about scientific results?

"Paper medium" is almost never used by readers. And if the function of "a medium" is still running, when it comes to distribution "speed" and "number of copies", it lags behind the digital medium. Moreover, from the "open" medium, a free access to which is provided in " $24 \times 7$ " mode. It is the "format", chosen by an increasing number of scientific journals [6].

What to do then with familiar, or as they say now, "offline" activities, such as congresses, conferences, and symposiums? In this case, it is possible to use not only usual "promotion formats" in the form of plenary and sectional reports, discussions and "round tables", but also new formats. For example, summaries should be submitted in the form of a professionally prepared presentation. At the same time, it is possible to represent new material in more detail in a similar presentation by placing a QR-code with a link to a relevant scientific social network. For example, for it, the authors of the present article use the capacities of ResearchGate. All three "peaks" in Fig. 4 were obtained after using a similar approach, combined with the simultaneous placement of the link to the material in a personal ribbon on social network Facebook.

Perhaps, we should consider such activity as establishment of a new approach to use all opportunities, offered by similar scientometric study [13]. In this case, it is possible to use results as parameters, affecting indicators of quality and educational process, if such an organization is an educational institution [17]. It may be worthwhile considering the need to create an appropriate "role" [18], which would involve help in promotion of results of scientific activity in "digital scientometric environment". It is necessary to handle both the articles, originally published in digital form, and previously issued. Accordingly, considering "scientific teams" as project teams [16], it is necessary to separate an appropriate functional for providing effective communication for interaction with both internal and external environment. At this, the internal environment in an extended sense of this term includes all employees, involved in the process of preparation, publication, distribution, access provision and archiving of scientific publications of corresponding subdivisions of an organization. External environment involves dissemination of scientific information beyond a "scientific team" and a corresponding institution, and, as a whole, beyond national boundaries. 


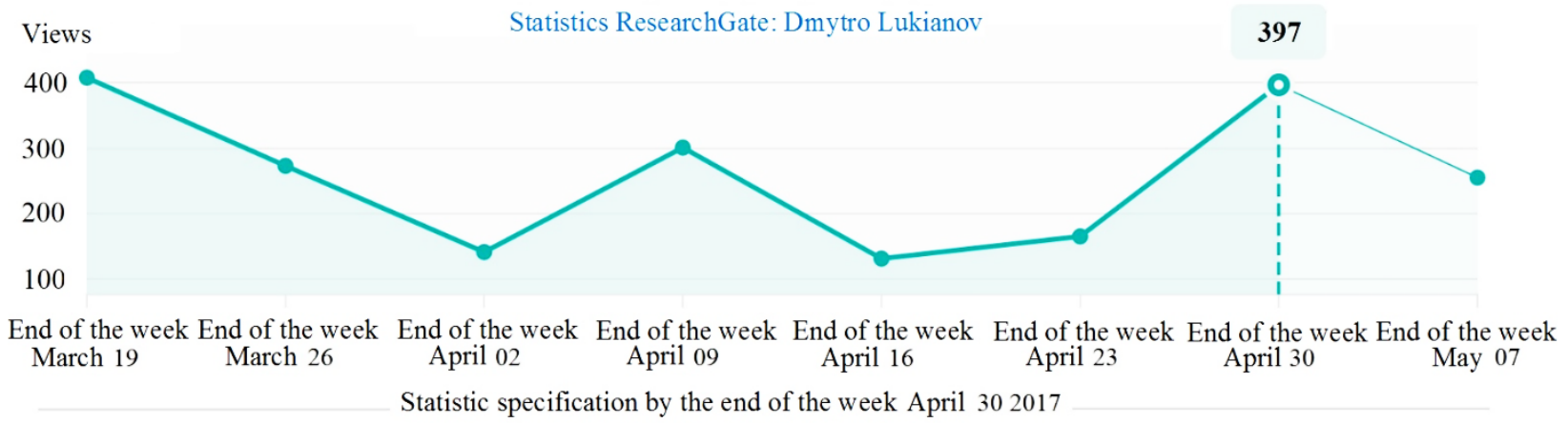

Fig. 4. Results of experiment on scientific content promotion using the social network Facebook

Thus, Principle 1 determines one of the objectives of scientific content promotion, involving readership expansion. In a hypothetical cognitive model (2), in order to expand readership, we will set magnitude of $\pi_{1.2}=0.60$. Obtained results of modeling represent a new structure of possible readership distribution (Fig. 5).

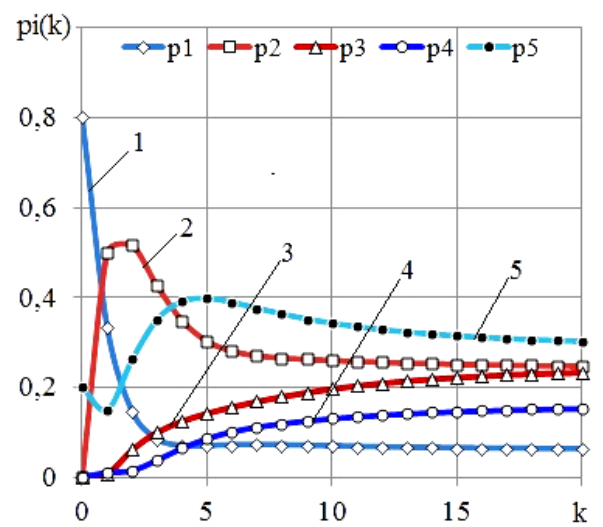

Fig. 5. A change in the probabilities of states on scientists' distribution by the level of attitude to a publication while expanding readership at $\pi_{1.2}=0.6: 1-$ unawareness; 2 - awareness; 3 - positive attitude; 4 - citing; 5 - negative attitude

Fig. 5 shows that the probability of citing publications increased to a magnitude of $\mathrm{p}_{4}(20) \approx 0.15$. The number of articles in the region of "unawareness" decreased by ten times to probability $\mathrm{p}_{1}(20) \approx 0.06$. The other states also underwent changing. In this case, probability of "negative attitude" $\mathrm{p}_{5}(20) \approx 0.3$ was almost 2 times as high as the percentage of publication citation. Thus, it can be concluded that principle 1 is acceptable for management of scientific communications.

Principle 2. "Content golden rule"

The authors of paper [12] derived a "content golden rule" with the optimal balance of different types of publications: $40 \%$ - involving; $30 \%$ - user's; $25 \%$-training; $5 \%$ - advertising. A disbalance towards publications of any type always affects effectiveness: either the audience is lost or business tasks are not resolved [12].

Article [20] describes the method, named after Nobel Laureate in Physics Richard Feynman. Feynman proposed a formula of training that allowed him to understand things better than others. A subject-matter, an object or a concept that needs to be examined do not make any difference. The method works everywhere. It is not only an excellent method of training, but also a window to an entirely different way of thinking.
The essence of Feynman's method lies in the utmost clarity of presenting complicating concepts and theories. According to Feynman, articles must be spelled out so clearly that even a schoolboy could understand their sense. It is on the basis of similar criteria that publications in the system Impactstory are assessed [21].

Thus, Principle 2 determines the problem of scientific content promotion, which involves clear, understandable, and distinct presentation of material of the article. In a hypothetical cognitive model (2), with a clear content representation, the region of "positive attitude" of readers' community increases, that is why we will accept the new value of $\pi_{2.3}=0.5$. In this case, parameter of readership expansion in accordance with Principle 1 will be set as $\pi_{1.2}=0.6$. The obtained results of modeling reflect a new structure of possible readership distribution (Fig. 6).

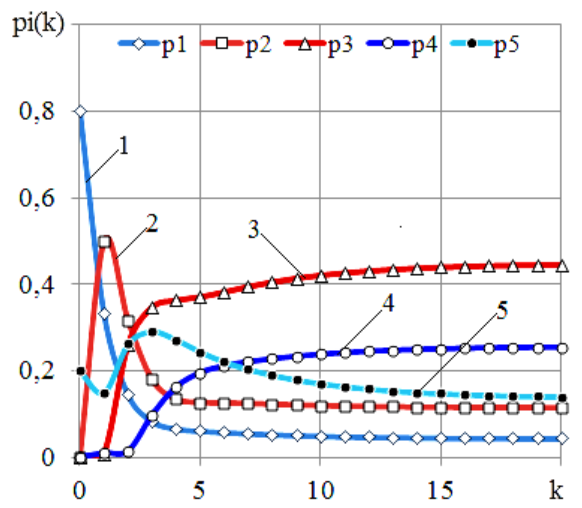

Fig. 6. A change in the probabilities of states on scientists' distribution by the level of attitude to a publication at readership expansion for $\pi_{1.2}=0.6$ and a provision of presentation clarity $\pi_{2.3}=0.5$ : 1 - unawareness; 2 - awareness; 3 - positive attitude; 4 - citing; 5 - negative attitude

As Fig. 6 shows, the system as a whole passed on to the region of positive attitude to a publication: $\mathrm{p}_{3}(20) \approx 0.45$. In this case, citation probability significantly increased: $\mathrm{p}_{4}(20) \approx 0.25$. At the same time, in comparison with data from Fig. 5, probability of negative attitude to publications decrease almost by two times and reached $\mathrm{p}_{5}(20) \approx 0.14$.

Principle 3. "Creation of unique content".

An example of best practices [12] shows that when implementing projects of content promotion on the Internet, being first is important: "The task of each team member is to find, apply, and teach others everything new and useful. It is 
important to optimize one's own work and be the first to do it. Competitors are awake".

In relation to a "digital" presentation of scientific content, this principle of "scientific SMM" is transformed into the rule: "Create and protect your uniqueness!" At a minimum, it is worth considering how to resolve the contradiction, that, on the one hand, one wants to get early feedback as for material of the article, and, on the other hand, there may be concerns about possible plagiarism [22].

Therefore, it is desirable to submit publications to journals that offer DOI [23], which is connected with an open access to digital copies of articles through CrossRef or DataCite. In this case, anti-plagiarism programs can detect borrowing of a text by other authors [22]. Thus, an article, which contains plagiarism, will not be published.

In a hypothetical cognitive model (2), the level of uniqueness of articles will be reflected in an increase in the "citation" segment in readers' community, that is why we will accept a new value of $\pi_{3.4}=0.7$. In doing so, we will leave the previous parameters of readership expansion $\left(\pi_{1.2}=0.6\right)$ and provision of text clarity $\left(\pi_{2.3}=0.5\right)$, forming readers' distribution, shown in Fig. 6. For the given conditions of management of the own content, we will get the results of modeling, which reflect a new structure of possible readership distribution (Fig. 7).

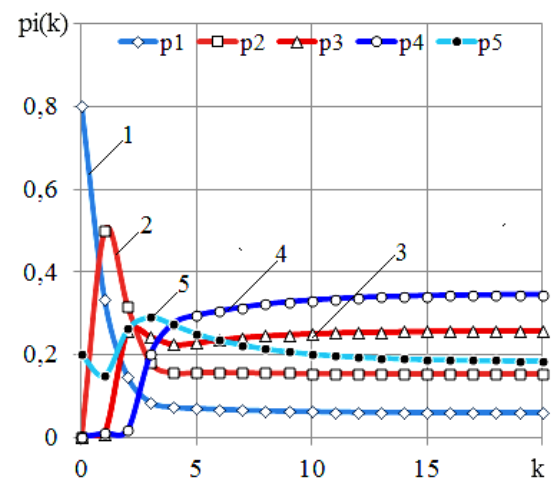

Fig. 7. A change in the probabilities of states of scientists' distribution by segments of attitude to a publication for parameters of system $-\pi_{1.2}=0.6, \pi_{2.3}=0.5$ and $\pi_{3.4}=0.7$ : 1 - unawareness; 2 - awareness; 3 - positive attitude; 4 - citing; 5 - negative attitude

As Fig. 7 shows, the citation segment $\mathrm{p}_{4}(20) \approx 0.35$ increased. The percentage of readers in the segment of positive attitude towards a publication decreased to magnitude $\mathrm{p}_{3}(20) \approx 0.25$. The remaining segments compared with data in Fig. 6 did not change significantly. Outcomes, shown in Fig. 7, demonstrate persuasively that uniqueness of articles and their scientific level have a major impact on citation indicators.

\section{Principle 4. "Client mania".}

The authors of [12] note that their client, in essence, is the most valuable of all that any company has. Here, both feedback speed and quality are essential. Communication is the basis of social networks. Communication with one's own audience is the basis for SMM.

Who are "Clients" in scientific SMM? Everyone who demonstrates interest in publications: conference participants, colleagues in research activities, staff, students, just participants in the professional community. Everybody may have intentions to obtain some materials from the authors
[25]. The same applies to "scientific SMM". It is possible to publish a document and provide access to an article as to a full-text document. Or you may place only a title and an abstract. You can provide full access upon request (or deny it). It will probably be the right idea to represent your articles not through e-mail/flash drive memory, but rather by reference to a requested document depositor in a personal ResearchGate profile. In ResearchGate, "responsiveness" is also measured by the number of times the access on request access for certain documents was granted. Emergence of such resource, as Impactstory [21], which uses the indicator of "degree of text perception ease", rewarding an author by appropriate message in case of such "accomplishment", became a kind of indicator of "client orientation".

Let us show how "client-oriented" work with readers will influence promotion of publications. It is clear that as a result of communications with readers, there may by questions concerning clarification of the application areas for research, described in the article. Expansions on the structure and parameters of the used models are possible. All this will ultimately help to reduce the segment of "negative attitude" to publications, which in the parameters of the model (2) will influence magnitude $\pi_{6.2}$. Let us take value of $\pi_{6.2}=0.5$ of a hypothetical cognitive model (2). All other parameters remain the same compared with the variant of the system, shown in Fig. 7.

For the given content management conditions, we will obtain results of modeling that reflect the readership distribution structure, taking into account "client orientation" (Fig. 8).

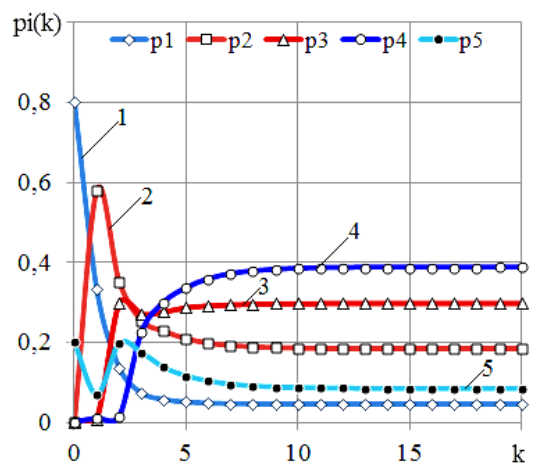

Fig. 8. A change in the probabilities of states of scientists' distribution by segments of attitude to a publication for parameters of system $-\pi_{1.2}=0.6, \pi_{2.3}=0.5, \pi_{3.4}=0.7$ and $\pi_{5.2}=0.5$ : 1 - unawareness; 2 - awareness; 3 - positive attitude; 4 - citing; 5 - negative attitude

As Fig. 8 shows, citation segment increased to $\mathrm{p}_{4}(20) \approx$ $\approx 0.4$. Percentage of readers in the segment of negative attitude to a publication decreased to magnitude $\mathrm{p}_{3}(20) \approx 0.08$. Volumes of the other segments compared with data from Fig. 7 did not change significantly. Outcomes, shown in Fig. 8, convincingly demonstrate that consideration of specifics of a subject-area of publications and customer orientation have a significant impact on promotion of publications and their citation.

Principle 5. "Honesty is an advantage in business".

There is one rule to follow, according to the authors of paper [12]. This rule is expressed in conscientious performance of work, objectivity of results reporting, and measurability of indicators.

Let us explore what impact "honest" work with readers will have on the promotion of publications. It is clear that 
reliable and objective data on a relevant and demanded subject-matter will be noticed by readers. References to these works, which will emerge as a result, will lead to an extended discussion and practical use of the results of an article. All this eventually will contribute to decreasing of "awareness" segment, which in the parameters of model (2) will influence magnitude $\pi_{4.2}$. Let us accept value $\pi_{4,2}=0.5$ for a hypothetical cognitive model (2). All other parameters will remain without changes compared with the variant of the system, represented in Fig.8.

After all introduced changes, we will obtain a matrix of transition probabilities:

$$
\left\|\pi_{i j}\right\|=\left\|\begin{array}{lllll}
0,39 & 0,60 & 0,01 & 0 & 0 \\
0 & 0,20 & 0,50 & 0 & 0,30 \\
0 & 0 & 0,30 & 0,70 & 0 \\
0,05 & 0,02 & 0,30 & 0,63 & 0 \\
0,10 & 0,50 & 0 & 0,05 & 0,35
\end{array}\right\| .
$$

Modelling with the use of the designed Markovian model for the variant of transition probabilities (4), that is, a new state, revealed results that are shown in Fig. 9.

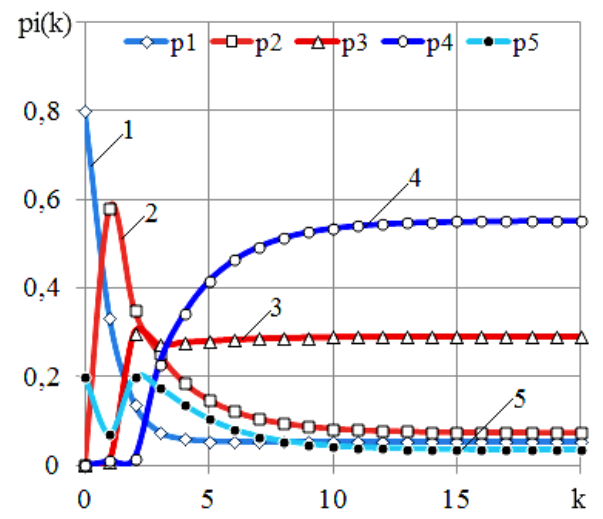

Fig. 9. A change in the probabilities of states of scientists' distribution by segments of attitude to a publication for parameters of system (4): 1 - unawareness; 2 - awareness;

3 - positive attitude; 4 - citing; 5 - negative attitude

As Fig. 9 shows, the citation segment $\mathrm{p}_{4}(20) \approx 0.55$ increased. Percentage of readers in the segment of positive attitude to a publication got established at the level of $\mathrm{p}_{3}(20) \approx$ $\approx 0.29$. Probability of negative attitude significantly decreased $\mathrm{p}_{5}(20) \approx 0.03$. The rest of the segments did not essentially change compared with the data in Fig. 8. Results, shown in Fig. 9, do not contradict the hypothesis that objectivity of results presentation and data measurability have a significant impact on citation indices.

\section{Discussion of results of managing social scientific networks}

Generalization and design of applied aspects of the Markov chains for application of SMM methodology expands possibilities of proactive career management of publication promotion in scientific environment [26].

We created a Markovian model, which represents a random process of cohort distribution of potential readers from $S_{1}$ - unawareness (Awareless) through $S_{2}$ - awareness
(Awareness) and $S_{3}-$ positive attitude (Attitude) to state $S_{4}$ - citation (Action). In this case, inclusion of state $S_{5}-$ negative attitude (Abort) leads to formation of a complete group. Probabilities of states of a complete group of incompatible events describe a random process, in which one of the events will certainly be realized [27].

Application of the Markov chains in scientific research is complicated by difficulty of "setting" a model to a particular system through determining the matrix of transition probabilities [28]. It was shown that values ${ }_{s}$ of transition probabilities can be determined based on characteristics of communications, taking into account time cost for performance of operations (Table 1). Such cognitive approach allows exploring the system in a wide range of changes in characteristics of states.

Using the designed Markovian model, it is possible to evaluate the influence of most of characteristics of the system on the course of a project. But a general conclusion, which can be drawn from findings of this study, is that an author's active participation in promotion of his publications in scientific environment determines a project's result.

The Markovian model of distribution of scientists' scientific community by the level of attitude to a publication makes it possible to represent random processes of "independent life" of articles depending on the number of communications (contacts) in social scientific networks. Similar studies have not been carried out before. With the use of the Markovian cognitive simulation model, we proved the hypothesis of fundamental possibility of communication management in social scientific networks to actualize publications in the world's scientific community. It was shown that the use of the analogy method in reference to promotion of findings of scientific research and promotion of information resources [12] on the Internet is justified.

In relation to scientific activity, it should be noted that all five principles of best practices, which were considered, appeared to be fully applicable. It is possible to recommend the following principles as primary principles for "scientific SMM":

- One is the field is not a warrior;

- Content balance;

- Create and protect your uniqueness;

- Client-orientation;

- Measurability.

The obtained results of the study of effectiveness of SMM application reflect the need for authors' participation in support of publications promotion in social networks.

It is rational to focus subsequent research in the area of enhancing effectiveness of operation in social scientific networks on designing theoretical methods of determining the elements of the matrix of transition probabilities. This will make it possible to scientifically determine the trajectory of development of publishing activity for the actualization and promotion of publications in the world's scientific community.

\section{Conclusions}

1. The structure of the stage of scientific publications lifecycle, specifying the levels of perception of articles by readers' community, was proposed. Possible states of community of readers of scientific publications at this stage include such levels of attitude: $S_{1}-$ Awareless (unawareness); 
$S_{2}$ - Awareness (awareness); $S_{3}-$ Attitude (positive attitude) $S_{4}$ - Action (citation); $S_{5}-$ Abort (negative attitude). Specified states characterize distribution of potential article's readers by groups of article perception. As communications in social networks develops, scientific community gains an opportunity to get acquainted with the content of articles and determine scientific and practical level of research findings, presented in the articles.

2. It is proposed to apply five basic principles of scientific content promotion, tested in the practice of promotion of information resources [12]. It was shown that projects of scientific content promotion on the Internet share characteristics with the activity involving promotion of information resources.

3. Specific features in the application of SMM methodology with the use of the designed Markovian model were explored. Directions of authors' activity on readership extension and provision of presentation quality were considered. It was shown that the level of articles' uniqueness, as well as "client-oriented" work with readers in the presence of reliable and objective information on a relevant and demanded subject-matter, will be noticed by readers. This will lead to an extended discussion and practical use of the article's findings. As a result, it is possible to expect references to these works to emerge. Ultimately, Management of submission of publications to social scientific networks will contribute to decrease segments $S_{1}$ - "unawareness" and $S_{2}$ - "awareness" with an increase in segments $S_{3}$ - "positive attitude" and $S_{4}-$ "citation". It was shown that authors' following up on their publications creates conditions for successful citations of publications in the world's scientific community.

\section{References}

1. Instagram [Electronic resource]. - Available at: https://www.instagram.com/ (Last accessed: 06.05.2017).

2. ResearchGate [Electronic resource]. - Available at: https://www.researchgate.net (Last accessed: 06.05.2017).

3. Mendeley [Electronic resource]. - Available at: https://www.mendeley.com (Last accessed: 06.05.2017)

4. Elsevier [Electronic resource]. - Available at: https://www.elsevier.com (Last accessed: 06.05.2017)

5. ORCID [Electronic resource]. - Available at: http://orcid.org (Last accessed: 06.05.2017)

6. Kukharchuk, Ye. O. Global scientometric system [Text] / Ye. O. Kukharchuk // Bibliotechnyi visnyk. - 2014. - Vol. 5. - P. 7-11. Available at: http://nbuv.gov.ua/UJRN/bv_2014_5_4

7. Hirsch, J. E. An index to quantify an individual's scienti c research [Text] / J. E. Hirsch // Proceedings of the National Academy of Sciences. - 2005. - Vol. 102, Issue 46. - P. 16569-16572. doi: 10.1073/pnas.0507655102

8. Google Scholar [Electronic resource]. - Available at: https://scholar.google.com.ua (Last accessed: 06.05.2017).

9. Academia.edu [Electronic resource]. - Available at: https://www.academia.edu/ (Last accessed: 06.05.2017).

10. Harzing, A.-W. The Publish or Perish Book [Text] / A.-W. Harzing. - Melbourne: Tarma Software Research Pty Ltd., 2010. 250 p. - Available at: https://harzing.com/publications/publish-or-perish-book

11. Biloshchytskyi, A. Evaluation methods of the results of scientific research activity of scientists based on the analysis of publication citations [Text] / A. Biloshchytskyi, A. Kuchansky, Y. Andrashko, S. Biloshchytska, O. Kuzka, O. Terentyev // Eastern European Journal of Enterprise Technologies. - 2017. - Vol. 3, Issue 2 (86). - P. 4-10. doi: 10.15587/1729-4061.2017.103651

12. Chulanova, E. (2017). Five of the principles SMM of LeadMachine [Electronic resource] / E. Chulanova. - Available at: http:// leadmachine.ru/2014/09/11/5-principov-smm-ot-leadmachine (Last accessed: 06.05.2017).

13. Goguns'kiy, V. D. General mechanisms of citation system of scientific articles [Text] / V. D. Goguns'kiy, V. O. Yakovenko, T. O. Lyashchenko, T. V. Otrads'ka // Bulletin of NTU "KhPI". Series: Strategic Management, Portfolio, Program and Project Management. 2016. - Vol. 5, Issue 1 (1173). - P. 14-18. doi: 10.20998/2413-3000.2016.1173.3

14. Rey, A. E. Memory plays tricks on me: Perceptual bias induced by memory reactivated size in Ebbinghaus illusion [Text] / A. E. Rey, G. T. Vallet, B. Riou, M. Lesourd, R. Versace // Acta Psychologica. - 2015. - Vol. 161. - P. 104-109. doi: 10.1016/j.actpsy. 2015.08.011

15. Gogunskii, V. Representation of project systems using the markov chain [Text] / V. Gogunskii, O. Kolesnikov, G. Oborska, A. Moskaliuk, K. Kolesnikova, S. Harelik et. al. // Eastern-European Journal of Enterprise Technologies. - 2017. - Vol. 2, Issue 3 (86). P. 60-65. doi: 10.15587/1729-4061.2017.97883

16. Kolesnikov, O. Development of the model of interaction among the project, team of project and project environment in project system [Text] / O. Kolesnikov, V. Gogunskii, K. Kolesnikova, D. Lukianov, T. Olekh // Eastern-European Journal of Enterprise Technologies. - 2016. - Vol. 5, Issue 9 (83). - P. 20-26. doi: 10.15587/1729-4061.2016.80769

17. Gogunskii, V. "Lifelong learning" is a new paradigm of personnel training in enterprises [Text] / V. Gogunskii, A. Kolesnikov, K. Kolesnikova, D. Lukianov // Eastern-European Journal of Enterprise Technologies. - 2016. - Vol. 4, Issue 2 (82). - P. 4-10. doi: 10.15587/1729-4061.2016.74905

18. Lukianov, D. Development the markovs model of the project as a system role communications team [Text] / D. Lukianov, K. Bespanska-Paulenko, V. Gogunskii, O. Kolesnikov, A. Moskaliuk, K. Dmitrenko // Eastern-European Journal of Enterprise Technologies. - 2017. - Vol. 3, Issue 3 (87). - P. 21-28. doi: 10.15587/1729-4061.2017.103231

19. Gogunskii, V. Developing a system for the initiation of projects using a Markov chain [Text] / V. Gogunskii, A. Bochkovsky, A. Moskaliuk, O. Kolesnikov, S. Babiuk // Eastern-European Journal of Enterprise Technologies. - 2017. - Vol. 1, Issue 3 (85). P. 25-32. doi: 10.15587/1729-4061.2017.90971

20. The Feynman method: three steps that allow you to quickly master any subject [Electronic resource]. - Available at: http://ideanomics.ru/articles/7981 (Last accessed: 06.05.2017). 
21. Impactstory blog [Electronic resource]. - Available at: http://blog.impactstory.org/ (Last accessed: 06.05.2017).

22. Lizunov, P. Detection of near duplicates in tables based on the locality-sensitive hashing and the nearest neighbor method [Text] $/$ P. Lizunov, A. Biloshchytskyi, A. Kuchansky, S. Biloshchytska, L. Chala // Eastern-European Journal of Enterprise Technologies. 2016. - Vol. 6, Issue 4 (84). - P. 4-10. doi: 10.15587/1729-4061.2016.86243

23. The DOI® System [Electronic resource]. - Available at: http://www.doi.org/ (Last accesed: 06.05.2017).

24. We are a not-for-profit membership organization for scholarly publishing working to make content easy to find, cite, link, and assess [Electronic resource]. - Available at: https://www.crossref.org/ (Last accessed: 06.05.2017).

25. Ivanova, V. P. (2012). Level character of the understanding of scientific text [Electronic resource] / V. P. Ivanova. - Available at: http://psyjournals.ru/files/54306/psyedu_2012_n3_Ivanova.pdf

26. ISO 21500:2012. Guidance on project management [Electronic resource]. - Available at: https://www.iso.org/standard/50003.html

27. Hunter, J. J. The computation of key properties of Markov chains via perturbations [Text] / J. J. Hunter // Linear Algebra and its Applications. - 2016. - Vol. 511. - P. 176-202. doi: 10.1016/j.laa.2016.09.004

28. Van der Hoorn, B. Playing projects: Identifying flow in the 'lived experience' [Text] / B. van der Hoorn // International Journal of Project Management. - 2015. - Vol. 33, Issue 5. - P. 1008-1021. doi: 10.1016/j.ijproman.2015.01.009

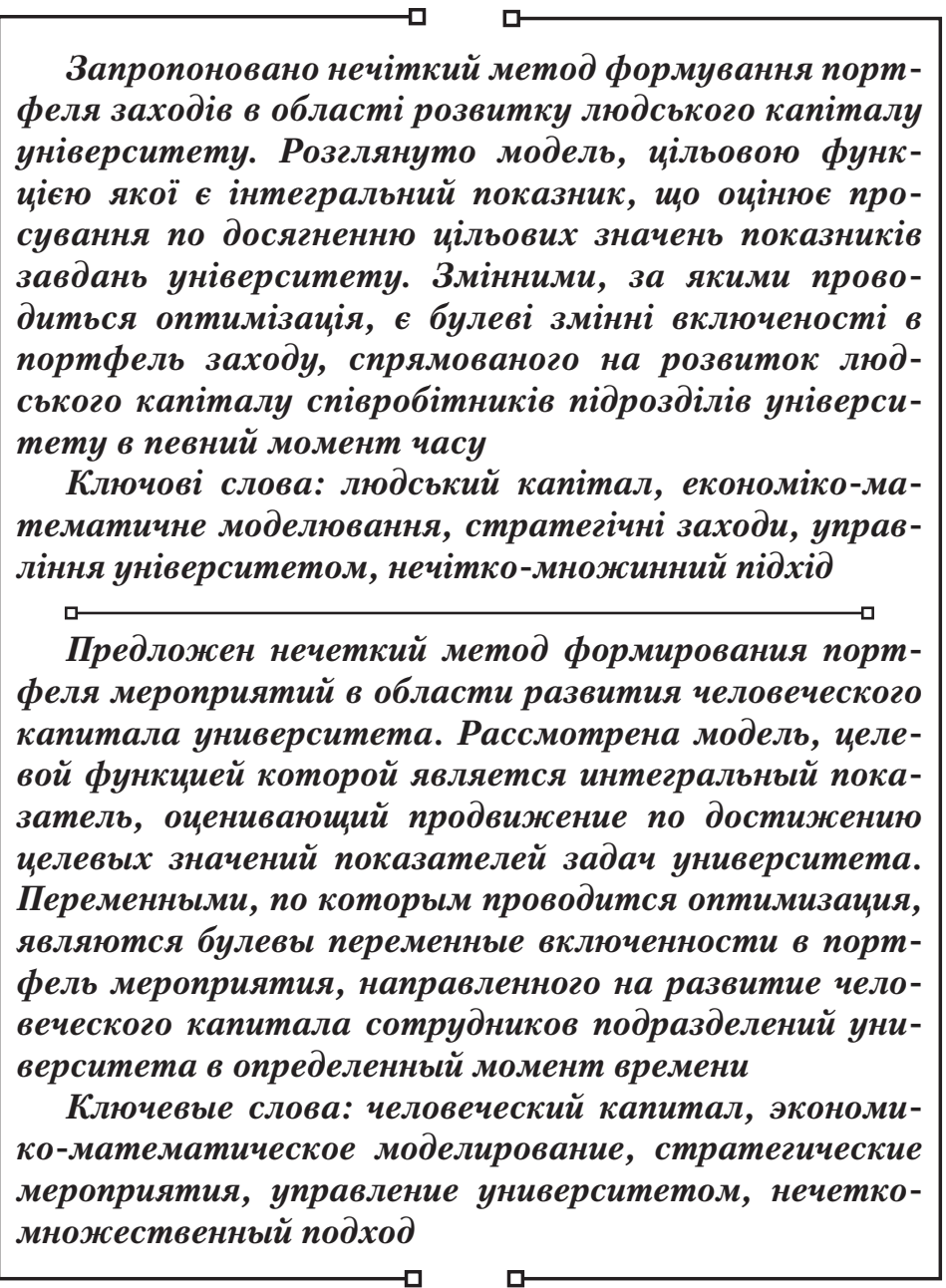

UDC 65.01

DOI: $10.15587 / 1729-4061.2017 .103979$

DEVISING A

FUZZY MODEL FOR COMPILING A PLAN OF ACTIVITIES AIMED AT DEVELOPING HUMAN CAPITAL IN UNIVERSITY

\author{
L. Mazelis \\ Doctor of Economic Sciences, \\ Head of Department* \\ E-mail: Lev.Mazelis@vvsu.ru \\ K. Lavreny u k \\ Assistant* \\ E-mail: Kirill.Lavrenyuk@vvsu.ru \\ Vladivostok State University of \\ Economics and Service
} *Department of Mathematics and Modeling

Gogolya str., 41, Vladivostok, Russia, 690014

\section{Introduction}

Managing sustainable development of a university is aimed at fulfilling of the tasks arising from strategic objectives and interrelated with the main indicators of the effectiveness of activity of universities. This is possible, among other factors, through competent and reasonable investment of available resources.

In this case, one of the priority areas of investment is the human capital of the university, which exerts decisive influence on the implementation of the university development strategy.
Human capital is one of the most important and, at the same time, very specific resource, which makes it rather difficult to assess effects that occur at a change in its magnitude. At present, world system of the higher education undergoes a transition from the translation of knowledge to a diversified university, whose basis of the educational process and economics is formed by research work and innovative activity. These changes further increase the importance of human capital and investment strategy in the field of its development.

Decomposition of strategic objectives to the level of structural divisions of the university leads to the necessity 